\title{
Preventing acute renal failure is crucial during acute tumor lysis syndrome
}

\author{
Michael Darmon, Guillaume Thiery, Elie Azoulay
}

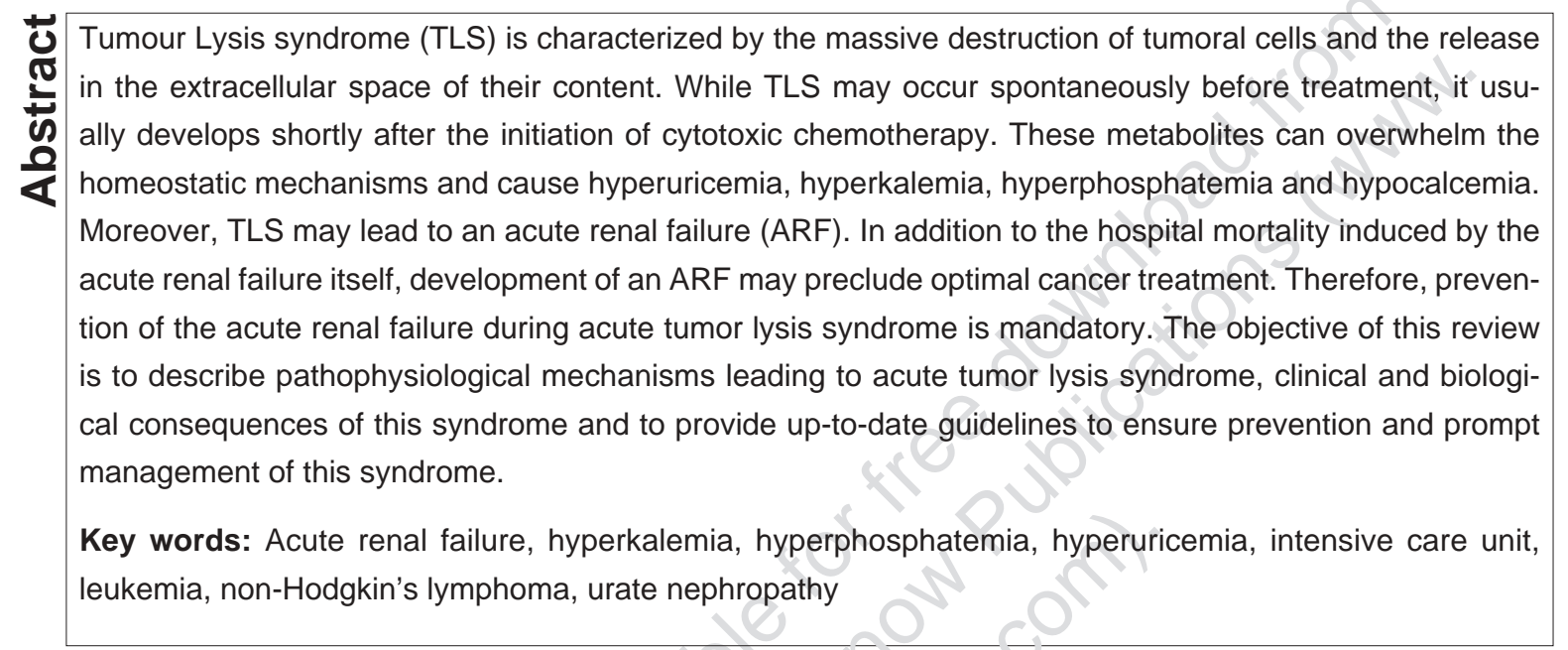

\section{Introduction}

If clinical infection remains the leading cause of organ failure in critically ill cancer patients (CICPs), several reports point out the increasing proportion of patients with cancer admitted into intensive care unit (ICU) with organ failure related to the malignancy. ${ }^{[1-3]}$ Some of these complications may be directly related to the extent of the malignancy. This may include, acute renal failure related to specific infiltration by non-Hodgkin's lymphoma or cancer, acute respiratory failure related to pulmonary leukemic infiltration or a bulky mediastinal tumor and coma that may be due to specific central nervous system involvement. ${ }^{[4-7]}$ Most of these specific organ failures will require initiation of cancer chemotherapy

From:

Assistance des Hôpitaux de Paris, Saint-Louis University Hospital, Medical Intensive Care Unit and University Paris VII, Paris, France (MD, GT, EA), Assistance des Hôpitaux de Paris, Creteil University Hospital, Medical Intensive Care Unit and University Paris XII, Creteil, France (MD)

\section{Correspondance:}

Dr. Michael Darmon, Service de réanimation médicale, Hôpital Henri Mondor. 51 avenue du Mal de Lattre de Tassigny, 94010 Creteil, France.

E-mail: michael.darmon@sls.ap-hop-paris.fr along with the initiation of organ support. Our team and Benoit et al already demonstrate that it can be performed allowing a hospital survival superior to $50 \% .^{[2,3]}$ Tumor Lysis syndrome (TLS) may occur spontaneously, as consequences of cancer chemotherapy initiation or even after a single dose of steroids. ${ }^{[8,9]}$ This syndrome is characterized by the massive destruction of tumoral cells and the release in the extracellular space of their content. ${ }^{[8,9]}$ Therefore, TLS may lead to the development of an acute renal failure which may, in itself, cause substantial morbidity and mortality. ${ }^{[10]}$ Indeed, in the critically ill cancer patients acute renal dysfunction is associated with mortality rates ranging from $72 \%$ to $85 \%$ when a renal replacement therapy is needed. ${ }^{[11,12]}$ Moreover, the association between TLS and acute renal failure will promote a dramatic increase of kaliemia and phosphatemia leading potentially to cardiac arrhythmia or sudden death. ${ }^{[8,9]}$ Last and in addition to the hospital mortality associated with ARF, development of an ARF may preclude optimal cancer treatment by requiring a 
decrease in chemotherapy dosage or by contraindicating potentially curative treatment. ${ }^{[13]}$

Therefore, prevention of acute renal failure during acute tumor lysis syndrome is mandatory. The objective of this review is to describe pathophysiological mechanisms leading to acute tumor lysis syndrome, to describe clinical and biological consequences of this syndrome and to provide up-to-date guidelines to ensure prevention and prompt management of TLS.

\section{Materials and Methods}

Search Strategy: Combinations of key words related to acute renal failure (e.g., acute renal failure, dialysis, hemofiltration, ICU...), cancer (cancer, malignancy, chemotherapy, bone marrow transplantation...) and TLS (acute tumor lysis syndrome, TLS, "Tumor Lysis syndrome" [MeSH], hyperuricemia, hyperphosphatemia, urate nephropathy, nephrocalcinosis [MeSH]) were used to search the MEDLINE database, OVID database and the Cochrane Group database. The last search was performed in April 06. We checked the bibliographies of retrieved reports and reviews. We carefully checked the reviews and articles focusing on acute kidney failure in the general ICU population and the articles focusing on the critically ill cancer patients.

Most relevant articles were selected by the authors (GT, MD and EA) in a way so as to give a concise and an up-to-date overview of the problem.

\section{Pathophysiology and Clinical Presentation}

TLS occurs as results of a massive destruction of malignant cells both spontaneously and after initiation of cancer chemotherapy. While TLS may occur spontaneously before treatment, it however usually develops shortly after the initiation of cytotoxic chemotherapy ${ }^{[14]}$ Critically ill cancer patients with specific organ failure could be an exception to that rule. We recently described a $31 \%$ incidence of spontaneous TLS manifestations at ICU admission in a selected population of critically ill cancer patients. ${ }^{[3]}$

Massive cells destruction will lead to a rapid release of intracellular anions, cations and metabolic products of proteins and nucleic acids into the bloodstream. ${ }^{[15]}$ As consequences of their high intracellular concentration, potassium, calcium, phosphates and uric acid will be released in the extracellular space. ARF may develop, the most common mechanism being uric acid crystal formation in the renal tubules secondary to hyperuricemia. Another cause may be calcium phosphate deposition related to hyperphosphatemia. While ARF leads to further increase in above describe metabolites, a vicious circle will therefore be initiated. Mechanisms and consequences of TLS are summarised in the Figure 1.

\section{Uric acid}

Malignant cells carry a large burden of nucleic acid products due to their high cellular activity and turnover. The release in the extracellular space of purine nucleic acid and their subsequent transformation into uric acid will lead to hyperuricemia. ${ }^{[16-18]}$ Renal handling of this urate load involves free filtration at the glomerulus, partial proximal tubular reabsorption and distal renal tubular secretion. ${ }^{[19,20]}$ Hyperuricemia is considered to be necessary for the development of urate nephropathy. ${ }^{[20]}$ Indeed, uric acid is only poorly soluble in water and may therefore lead to crystals deposit. However, there is only a relatively poor correlation between the plasma urate levels and degree of renal dysfunction suggesting that additional factors may modify the rate of urate precipitation and the severity of the subsequent renal damages. ${ }^{[21]}$ At least two additional mechanisms can be involved in this process. First, the uric acid pKa (5.4-5.7) is responsible of a further decrease in its solubility in presence of an acidic $\mathrm{pH}^{\left[{ }^{[22]}\right.}$ Urinary $\mathrm{pH}$ may have therefore accounted for the lack of correlation between urate level and ARF development. Second, variations in urinary flow are associated with high variations of tubular concentration of uric acid. ${ }^{[21]} \mathrm{In}$ presence of a low urinary flow and of an acidic $\mathrm{pH}$, the threshold at which uric acid precipitates into crystals may

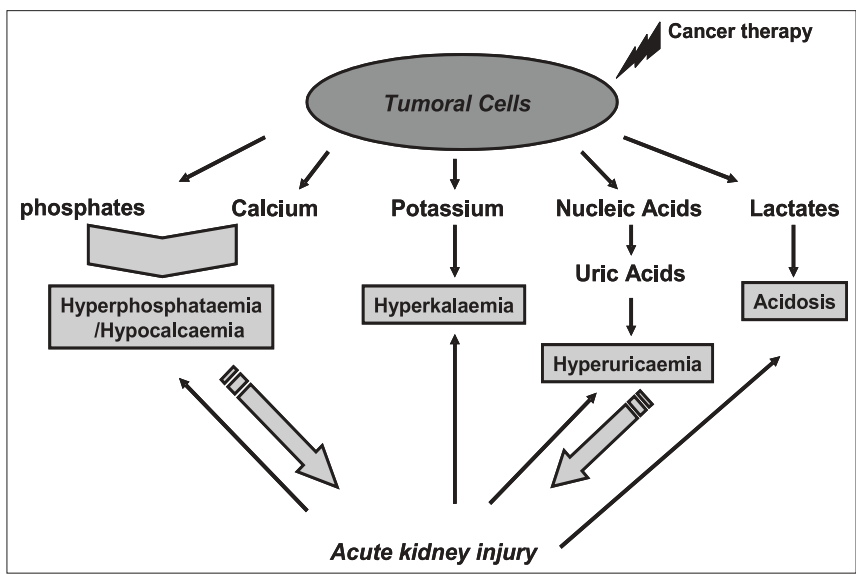

Figure 1: Pathophysiology of the tumor lysis syndrome 
be reached leading to tubular obstruction. ${ }^{[21]}$

\section{Hyperphosphatemia and hypocalcemia}

Cell death will lead to the release of degenerated nuclear material, including nucleotides and phosphate. Malignant cells may contain as much as four times the intracellular phosphorous contained in a mature lymphocyte. ${ }^{[23]}$ This large burden may saturate the renal capacity to excrete it. This phenomenon will be amplified in presence of a decrease urinary flow. The phosphates concentration will therefore raise leading to precipitation of calcium-phosphate crystals. These crystals will lead to nephrocalcinosis, urinary obstruction and tissue deposits. As consequences of the hyperphosphatemia and calcium-phosphates precipitation, hypocalcemia may appear. This hypocalcemia is only rarely symptomatic and do not require any treatment if asymptomatic.

Calcium phosphate crystal deposition has been reported to occur when the [Calcium] X [Phosphates] molar product exceed 4.6. ${ }^{[24]}$ Nevertheless, the study suggesting this threshold had however number of methodological weaknesses and this cut-off must be interpreted with caution. In addition, hypocalcemia that appears as consequence of the calcium phosphate deposition will lead to an underestimation of the [Calcium] $\mathrm{X}$ [Phosphates] molar product.

\section{Hyperkalemia}

Potassium is known to be a predominantly intracellular ion. The large burden of potassium released as consequences of the cells destruction may overwhelm the renal excretion ability and lead to a hyperkalemia. Moreover, the potential acute renal failure or acidosis associated with the TLS may exacerbate this hyperkalemia. Last, it has been supposed that stress due to radiotherapy or chemotherapy may reduce ATP levels, resulting in leakage of potassium out of the tumor cells before complete lysis and therefore to an early peak in serum potassium concentration. ${ }^{[15]}$

\section{Acidosis}

Lactic acidosis as been retrieved in association with this syndrome and its extent is correlated with the severity of TLS. ${ }^{25]}$ Pathophysiological mechanisms leading to this lactic acidosis are probably multiple, including hepatic failure and tumor ischemia resulting from the poor neovascularization of the tumors. ${ }^{[26]}$ However, it has been recently demonstrated that lactic acidosis can be caused by the loss of mitochondrial membrane potential during apoptosis. ${ }^{[25]}$ Therefore, massive apoptosis of a tumor mass during cancer chemotherapy may lead to a lactic acidosis and may be a pathological event of the tumor lysis syndrome.

In addition, the high phosphates concentration in serum, in itself, provokes an additional metabolic acidosis.

\section{Clinical presentation}

Although TLS may be responsible of several symptoms, the biological abnormalities usually remain the preeminent consequences of this syndrome. Acute renal failure can lead to fluid overload and pulmonary edema; Hyperkalemia or hyperphosphatemia may lead to cardiac arrhythmia or sudden death; and last, calcium and phosphate abnormalities may lead to infrequent muscle cramps or seizures. However, in the great majority of the patients, these symptoms are the markers of very advanced tumor lysis syndrome and must be considered as a failure to prevent it or treat it aggressively. In addition to these clinical and life-threatening consequences, this delay will have an impact on the ability to offer complete remission to these patients, via the development of an acute renal failure. ${ }^{[13]}$ Our aim should therefore be to identify patients at risk of acute TLS, in way to prevent or to treat TLS aggressively.

\section{Risk Stratification}

Early recognition of patients at high risk for TLS is mandatory and allows the initiation of prophylactic measures. TLS is classically described in patients with extensive, rapidly growing, chemosensitive tumours. This syndrome typically occurs in patients with high-grade hematological malignancies, especially high grade nonHodgkin's lymphoma or both acute myeloid and acute lymphoid leukemia. ${ }^{14,23,27-30]}$ Classical risk factors include large tumor burdens, lactate dehydrogenase levels above $1500 \mathrm{IU}$, extensive bone marrow involvement and high tumor sensitivity to chemotherapeutic agents. ${ }^{[28]}$ TLS has also been reported in patients with fast-growing solid tumours such as testicular cancer.$^{[15,31]}$ Additionally, several low grade hematological malignancies including chronic lymphoid lymphoma, solid tumors or myeloma have been described to be associated with TLS. ${ }^{[32-36]}$ This may be at least partially due to the increasing efficiency of novel anticancer therapies. Several of the new 
treatments (namely rituximab, bortezumib, thalidomide, tamoxifen or interferon $\alpha$ ) have been associated with the development of TLS in low grade malignancies. ${ }^{[34-39]}$ The wide spectrum of situations associated with TLS is listed in the Table 1.

\section{Prevention and Treatment}

The goal of the TLS treatment is to prevent acute renal failure, which may enhance dramatically TLS biological and clinical consequences. Since the development of a hyperkalemia remains uncommon in the absence of acute renal failure, two primary end-points can be delineated: the control of hyperuricemia and the prevention of nephrocalcinosis. If acute renal failure develops despite prevention, extra-renal therapy should be initiated quickly, aimed at clearance of uric acid and phosphate in way to limit further kidney impairment. ${ }^{[40]}$ In addition, hypophosphatemia and hypokalemia might be present before cancer chemotherapy initiation. These abnormalities give evidence of a high tumour burden and of a high risk of TLS and should therefore not be corrected. Preventive measures are summarized in the Table 2.

\section{Fluid expansion}

Treatment's cornerstone remains the aggressive hydration through isotonic saline and the maintenance of a high urinary output allowing the urinary elimination of both uric acid and phosphates. ${ }^{[8,21,40]}$ Moreover the volume expansion will decrease uric acid, phosphates and potassium serum concentrations..$^{[9]}$ If urinary output decreases despite adequate fluid intakes, diuretics have been proposed, sometime in addition with mannitol. ${ }^{[8]}$ However, in our experience, diuretics are only poorly effective. Moreover, development of an oliguria indicates an acute renal failure and diuretics may delay the initiation of renal replacement therapy. Lastly, use diuretics may be far from innocuous in ARF ${ }^{[41]}$ We therefore believe that development of an oliguria despite fluid therapy might be a signal for the physician and leads to initiation of renal replacement therapy each time possible.

Urine alkalinization has initially been recommended, in way to promote urinary elimination of urate. ${ }^{[42]}$ This treatment is nowadays controversial for three reasons. First, the availability of recombinant urate oxidase therapy considerably reduces the risk of urate nephropathy. ${ }^{[30,43]}$ Second, urine alkalinization may induce calcium phosphate deposition. ${ }^{[4,45]}$ And last, it has been longrecognized that a high tubular fluid flow is the primary mechanism of protection in acute urate nephropathy, when urine alkalinization seems to play only a minor preventive role. ${ }^{[21]}$ Therefore, we believe that this poorly effective and potentially harmful treatment should not be recommended routinely anymore.

\section{Hypouricemic agents}

In addition to the hydration, several hypouricemic agents may reduce uric acid levels. Nonrecombinant urate oxidase (Uricozyme ${ }^{\circledR}$ ), available in Europe from 1975 to 2002, was very effective but has been associated with high rate of allergic reactions. More recently, recombinant urate oxidase (Rasburicase) has been shown to reduce uric acid levels, thereby diminishing the risk of uric acid deposition nephropathy. ${ }^{[30,43]}$ This agent transforms the uric acid into a compound five to 10 times more soluble in the urine: the allantoin..$^{[8]}$ Recombinant urate oxydase has been shown to decrease median uric

\section{Table 1: Risk factors of acute Tumors Lysis syndrome}

\section{High risk malignancies}

- High-grade non-Hodgkin lymphoma

- Acute lymphoid leukaemia

- Acute myeloid leukaemia

\section{Intermediate and low risk}

Intermediate risk:

- Myeloma ${ }^{[33,34,36]}$

- Low-grade non-Hodgkin

Lymphoma $^{[48]}$

- Small-cell lung carcinoma ${ }^{[31]}$

Low risk:

- Medulloblastoma ${ }^{[44]}$

- Breast or gastrointestinal carcinoma $^{[31]}$

\section{Anecdotal reports \\ - Rhabdomyosarcoma ${ }^{[31]}$ \\ - Vulvar carcinoma ${ }^{[31]}$ \\ - Ovarian carcinoma ${ }^{[31]}$ \\ - Thymoma ${ }^{[15]}$ \\ - Soft tissue sarcomas ${ }^{[31]}$}

- Metastatic seminoma ${ }^{[15]}$

- Melanoma[39]

- Prostatic neoplasm ${ }^{[49]}$

- Hepatoblastoma ${ }^{[31]}$

- Hepatocarcinoma ${ }^{[37,50]}$

- Colonic carcinoma ${ }^{[50]}$

- Pheochromocytoma ${ }^{[50]}$
Other risk factors

- Tumor spread

- Rapid tumor growth

- Chemosensitive tumor

- Lactates dehydrogenase $>1500 \mathrm{IU} / \mathrm{L}$

- Hypokalaemia/ hypophosphataemia before treatment

- Preexisting renal failure 
Table 2: Prevention and treatments of the Tumour Lysis syndrome

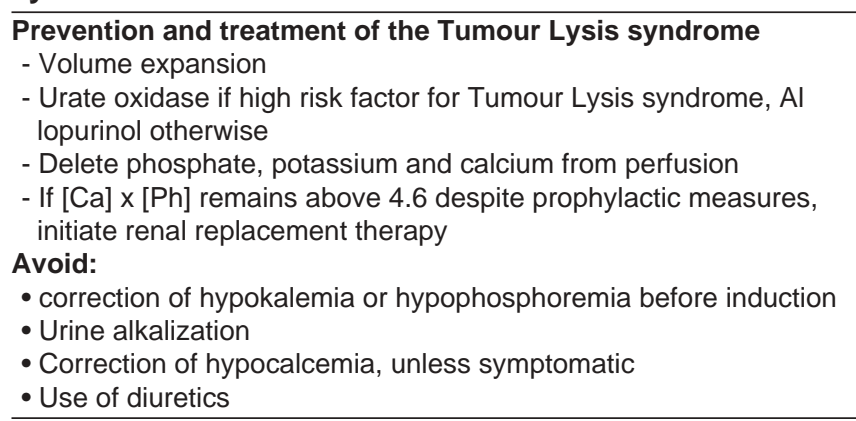

acid concentration from 577 to $60 \mu \mathrm{mol} / \mathrm{l}$ within four hours of therapy. ${ }^{[29]}$ Moreover, Recombinant urate oxydase has been shown to reduce significantly uric acid exposure time when compared to allopurinol. ${ }^{[30]}$ Last, used in a population of adult patients with aggressive nonHodgkin's lymphoma, Recombinant urate oxydase allowed the control of plasma uric acid within $4 \mathrm{~h}$ of injection and none of the patients developed hyperuricemia throughout the observation period. ${ }^{[43]}$ In addition, in the same study, no patient experienced acute renal failure. ${ }^{[43]}$ Although very effective, the recombinant urate oxydase is however also very expensive and its use should be limited to the prevention of TLS in high risk patients or treatment of established tumor lysis syndrome. ${ }^{[46]}$ Moreover, Rasburicase is contraindicated in patients with glucose-6phosphate dehydrogenase (G6PD) deficiency. ${ }^{[47]}$ Indeed, recombinant urate oxydase causes excessive hydrogen peroxide production when breaking down uric acid and may lead to hemolysis in such patients. ${ }^{[4]}$

In patients with low or intermediate TLS risk, allopurinol can be used as hypouricemic agent. Allopurinol is a xanthine analogue that will decrease transformation of xanthine into uric acid. ${ }^{[30]}$ However, if it may limit the risk of urate nephropathy in some patients, Allopurinol will also induce an increased xanthine and hypoxanthine serum concentrations. The solubility of these compounds is lower than that of uric acid and xanthine nephropathy may therefore develop. ${ }^{[45]}$ This complication is however uncommon and allopurinol is still indicated in this population.

\section{Prevention of nephrocalcinosis}

The prevention of nephrocalcinosis relies on the treatment of hyperphosphatemia and the eviction of any calcium therapy, except for the uncommon manifestations of hypocalcaemia. Concerning the treatment of hyperphosphatemia, only few treatments can be proposed in addition to hydration. Oral phosphates binders have been proposed but are only poorly effective. The persistence of a hyperphosphatemia despite saline infusion should lead to initiate renal replacement therapy. Moreover, it is crucial for the physician to keep in mind that the coexistence of a hyperphosphatemia and of a hypocalcemia is the signature of calcium phosphate crystals deposition.

\section{Indication and timing of the renal replacement therapies}

We believe that renal replacement therapy should be started on an emergency basis when hydration fails to produce a prompt metabolic improvement or when ARF develops. In addition to metabolic control, renal replacement therapy allows to protect kidney from further injuries. Phosphate clearance is higher with sequential dialysis that with hemofiltration but is frequently associated with a rebound effect after dialysis. ${ }^{[9]}$ Moreover, hemofiltration might be unable to produce an efficient metabolic control during the most severe TLS. Therefore, extended daily dialysis or isolated sequential dialysis followed by continuous hemofiltration should be the standard of care for TLS requiring renal replacement therapy. A study is currently ongoing to precise pharmacokinetic of cancer chemotherapies during renal replacement therapy.

\section{Conclusion}

TLS is a frequent and a life-threatening complication of the newly diagnosed malignancies. Both development of an acute renal failure or metabolic derangements may lead to ICU admission. It is critical for the physician in charge of the cancer patients to be able to recognize patients at high risk of developing TLS and to prevent it. Although the availability of uricolytic treatments has dramatically reduced the risk of urate nephropathy, calcium-phosphates crystals deposition may lead to acute renal failure and limit further cancer treatments. Therefore, when prevention measures do not allow a fast metabolic control, it is crucial to start renal replacement therapy on an emergency basis in way to allow an adequate renal protection. Further studies are needed to determine the optimal timing and modalities of the renal replacement therapy and to re-evaluate the risk factors of TLS. 


\section{References}

1. Azoulay E, Afessa B. The intensive care support of patients with malignancy: Do everything that can be done. Intensive Care Med 2006;32:3-5.

2. Benoit DD, Depuydt PO, Vandewoude KH, Offner FC, Boterberg $\mathrm{T}$, De Cock CA, et al. Outcome in severely ill patients with hematological malignancies who received intravenous chemotherapy in the intensive care unit. Intensive Care Med 2006;32:93-9.

3. Darmon M, Thiery G, Ciroldi M, de Miranda S, Galicier L, Raffoux $\mathrm{E}$, et al. Intensive care in patients with newly diagnosed malignancies and a need for cancer chemotherapy. Crit Care Med 2005;33:2488-93.

4. Azoulay E, Fieux F, Moreau D, Thiery G, Rousselot P, Parrot A et al. Acute monocytic leukemia presenting as acute respiratory failure. Am J Respir Crit Care Med 2003;167:1329-33.

5. Glimelius I, Molin D, Amini RM, Gustavsson A, Glimelius B, Enblad G. Bulky disease is the most important prognostic factor in Hodgkin lymphoma stage IIB. Eur J Haematol 2003;71:327-33.

6. Jackson N, Reddy SC, Harun MH, Quah SH, Low HC. Macular haemorrhage in adult acute leukaemia patients at presentation and the risk of subsequent intracranial haemorrhage. $\mathrm{Br} \mathrm{J}$ Haematol 1997;98:204-9.

7. Tornroth T, Heiro M, Marcussen N, Franssila K. Lymphomas diagnosed by percutaneous kidney biopsy. Am J Kidney Dis 2003;42:960-71

8. Cairo MS, Bishop M. Tumour lysis syndrome: New therapeutic strategies and classification. Br J Haematol 2004;127:3-11.

9. Davidson MB, Thakkar S, Hix JK, Bhandarkar ND, Wong A, Schreiber MJ. Pathophysiology, clinical consequences and treatment of tumor lysis syndrome. Am J Med 2004;116:546-54.

10. Metnitz PG, Krenn CG, Steltzer H, Lang T, Ploder J, Lenz K, et al. Effect of acute renal failure requiring renal replacement therapy on outcome in critically ill patients. Crit Care Med 2002;30:2051-8.

11. Lanore JJ, Brunet F, Pochard F, Bellivier F, Dhainaut JF, Vaxelaire JF et al. Hemodialysis for acute renal failure in patients with hematologic malignancies. Crit Care Med 1991;19:346-51.

12. Benoit DD, Hoste EA, Depuydt PO, Offner FC, Lameire NH, Vandewoude $\mathrm{KH}$, et al. Outcome in critically ill medical patients treated with renal replacement therapy for acute renal failure: Comparison between patients with and those without haematological malignancies. Nephrol Dial Transplant 2005;20:552-8.

13. Munker R, Hill U, Jehn U, Kolb HJ, Schalhorn A. Renal complications in acute leukemias. Haematologica 1998;83:416-21.

14. Altman A. Acute tumor lysis syndrome. Semin Oncol 2001;28:3-8.

15. Yarpuzlu AA. A review of clinical and laboratory findings and treatment of tumor lysis syndrome. Clin Chim Acta 2003;333:13-8.

16. Seegmiller JE, Laster L, Howell RR. Biochemistry of uric acid and its relation to gout. N Engl J Med 1963;268:821-7.
17. Seegmiller JE, Laster L, Howell RR. Biochemistry of uric acid and its relation to gout. N Engl J Med 1963;268:764-73.

18. Seegmiller JE, Laster L, Howell RR. Biochemistry of uric acid and its relation to gout. N Engl J Med 1963;268:712-6.

19. Gutman $A B, Y u T F$, Berger $L$. Tubular secretion of urate in man. J Clin Invest 1959;38:1778-81.

20. Berliner RW, Hilton JG, Yu TF, Kennedy TJ. The renal mechanism for urate excretion in man. J Clin Invest 1950;29:396-401.

21. Conger JD, Falk SA. Intrarenal dynamics in the pathogenesis and prevention of acute urate nephropathy. J Clin Invest 1977;59:78693.

22. Klinenberg JR, Goldfinger SE, Seegmiller JE. The effectiveness of the xanthine oxidase inhibitor allopurinol in the treatment of gout. Ann Intern Med 1965;62:639-47.

23. Flombaum CD. Metabolic emergencies in the cancer patient. Semin Oncol 2000;27:322-34

24. Hebert LA, Lemann J Jr, Petersen JR, Lennon EJ. Studies of the mechanism by which phosphate infusion lowers serum calcium concentration. J Clin Invest 1966;45:1886-94.

25. Tiefenthaler M, Amberger A, Bacher N, Hartmann BL, Margreiter $\mathrm{R}$, Kofler $\mathrm{R}$, et al. Increased lactate production follows loss of mitochondrial membrane potential during apoptosis of human leukaemia cells. Br J Haematol 2001;114:574-80.

26. Lettieri CJ, Berg BW. Clinical features of non-Hodgkins lymphoma presenting with acute liver failure: A report of five cases and review of published experience. Am J Gastroenterol 2003;98:1641-6.

27. Jeha S. Tumor lysis syndrome. Semin Hematol 2001;38:4-8.

28. Cohen LF, Balow JE, Magrath IT, Poplack DG, Ziegler JL. Acute tumor lysis syndrome. A review of 37 patients with Burkitt's lymphoma. Am J Med 1980;68:486-91.

29. Pui $\mathrm{CH}$. Urate oxidase in the prophylaxis or treatment of hyperuricemia: The United States experience. Semin Hematol 2001;38:1321.

30. Goldman SC, Holcenberg JS, Finklestein JZ, Hutchinson R, Kreissman $\mathrm{S}$, Johnson $\mathrm{FL}$, et al. A randomized comparison between rasburicase and allopurinol in children with lymphoma or leukemia at high risk for tumor lysis. Blood 2001;97:2998-3003.

31. Kalemkerian GP, Darwish B, Varterasian ML. Tumor lysis syndrome in small cell carcinoma and other solid tumors. Am J Med 1997;103:363-7.

32. Hussain K, Mazza JJ, Clouse LH. Tumor lysis syndrome (TLS) following fludarabine therapy for chronic lymphocytic leukemia (CLL): Case report and review of the literature. Am J Hematol 2003;72:212-5

33. Fassas AB, Desikan KR, Siegel D, Golper TA, Munshi NC, Barlogie $\mathrm{B}$, et al. Tumour lysis syndrome complicating high-dose treatment in patients with multiple myeloma. Br J Haematol 1999;105:938-41.

34. Terpos E, Politou M, Rahemtulla A. Tumour lysis syndrome in 
multiple myeloma after bortezomib (VELCADE) administration. J Cancer Res Clin Oncol 2004;130:623-5.

35. Yang $\mathrm{H}$, Rosove MH, Figlin RA. Tumor lysis syndrome occurring after the administration of rituximab in lymphoproliferative disorders: High-grade non-Hodgkin's lymphoma and chronic lymphocytic leukemia. Am J Hematol 1999;62:247-50.

36. Cany L, Fitoussi O, Boiron JM, Marit G. Tumor lysis syndrome at the beginning of thalidomide therapy for multiple myeloma. J Clin Oncol 2002;20:2212.

37. Lee CC, Wu YH, Chung SH, Chen WJ. Acute tumor lysis syndrome after thalidomide therapy in advanced hepatocellular carcinoma. Oncologist 2006;11:87-8.

38. Cech P, Block JB, Cone LA, Stone R. Tumor lysis syndrome after tamoxifen flare. N Engl J Med 1986;315:263-4.

39. Stoves J, Richardson D, Patel H. Tumour lysis syndrome in a patient with metastatic melanoma treated with biochemotherapy. Nephrol Dial Transplant 2001;16:188-9.

40. Humphreys BD, Soiffer RJ, Magee CC. Renal failure associated with cancer and its treatment: An update. J Am Soc Nephrol 2005;16:151-61.

41. Mehta RL, Pascual MT, Soroko S, Chertow GM; PICARD Study Group. Diuretics, mortality and nonrecovery of renal function in acute renal failure. JAMA 2002;288:2547-53.

42. Ten Harkel AD, Kist-Van Holthe JE, Van Weel M, Van der Vorst MM. Alkalinization and the tumor lysis syndrome. Med Pediatr Oncol 1998;31:27-8.

43. Coiffier B, Mounier N, Bologna S, Ferme C, Tilly H, Sonet A, et al. Efficacy and safety of rasburicase (recombinant urate oxidase) for the prevention and treatment of hyperuricemia during induction chemotherapy of aggressive non-Hodgkin's Iymphoma: Results of the GRAAL1 (Groupe d'Etude des Lymphomes de l'Adulte Trial on Rasburicase Activity in Adult Lymphoma) study. J Clin Oncol 2003;21:4402-6.

44. Baeksgaard L, Sorensen JB. Acute tumor lysis syndrome in solid tumors- a case report and review of the literature. Cancer Chemother Pharmacol 2003;51:187-92.

45. Haas M, Ohler L, Watzke H, Bohmig G, Prokesch R, DrumI W. The spectrum of acute renal failure in tumour lysis syndrome. Nephrol Dial Transplant 1999;14:776-9.

46. Annemans L, Moeremans K, Lamotte M, Garcia Conde J, van den Berg $\mathrm{H}$, Myint $\mathrm{H}$, et al. Pan-European multicentre economic evaluation of recombinant urate oxidase (rasburicase) in prevention and treatment of hyperuricaemia and tumour lysis syndrome in haematological cancer patients. Support Care Cancer 2003;11:249-57.

47. Browning LA, Kruse JA. Hemolysis and methemoglobinemia secondary to rasburicase administration. Ann Pharmacother 2005;39:1932-5.

48. Jensen M, Winkler U, Manzke O, Diehl V, Engert A. Rapid tumor lysis in a patient with B-cell chronic lymphocytic leukemia and lymphocytosis treated with an anti-CD20 monoclonal antibody (IDEC-C2B8, rituximab). Ann Hematol 1998;77:89-91.

49. Tanvetyanon T, Choudhury AM. Fatal acute tumor lysis syndrome, hepatic encephalopathy and flare phenomenon following combined androgen blockade. J Urol 2004;171:1627.

50. Vaisban E, Braester A, Mosenzon O, Kolin M, Horn Y. Spontaneous tumor lysis syndrome in solid tumors: Really a rare condition? Am J Med Sci 2003;325:38-40.

Source of Support: Nil, Conflict of Interest: None declared.

\section{Author Help: Online Submission of the Manuscripts}

Articles can be submitted online from http://w ww.journalonw eb.com. For online submission articles should be prepared in two files (first page file and article file). Images should be submitted separately.

1) First Page File:

Prepare the title page, covering letter, acknowledgement, etc., using a word processor program. All information which can reveal your identity should be here. Use text/rtf/doc/pdf files. Do not zip the files.

2) Article file:

The main text of the article, beginning from Abstract till References (including tables) should be in this file. Do not include any information (such as acknow ledgement, your names in page headers, etc.) in this file. Use text/rtf/doc/pdf files. Do not zip the files. Limit the file size to $400 \mathrm{~kb}$. Do not incorporate images in the file. If file size is large, graphs can be submitted as images separately without incorporating them in the article file to reduce the size of the file.

3) Images:

Submit good quality colour images. Each image should be less than $\mathbf{4 0 0} \mathbf{~ k b}$ in size. Size of the image can be reduced by decreasing the actual height and width of the images (keep up to about 4 inches) or by reducing the quality of image. All image formats (jpeg, tiff, gif, bmp, png, eps, etc.) are acceptable; jpeg is most suitable. The image quality should be good enough to judge the scientific value of the image. Always retain a good quality, high resolution image for print purpose. This high resolution image should be sent to the editorial office at the time of sending a revised article.

4) Legends:

Legends for the figures/images should be included at the end of the article file. 